\title{
Precision pointing of imaging spacecraft using gyro-based attitude reference with horizon sensor updates
}

\author{
N VENKATESWARAN $^{1}$, P S GOEL ${ }^{2}$, M S SIVA $^{3}$, P NATARAJAN ${ }^{3}$, \\ E KRISHNAKUMAR ${ }^{3}$ and N K PHILIP ${ }^{3}$ \\ ${ }^{1}$ Electronics and Photonics System Technology, GE John F Welch Technology \\ Center, Bangalore 560 066, India \\ ${ }^{2}$ ISRO Satellite Centre, Bangalore 560 017, India \\ ${ }^{3}$ Control Systems Group, ISRO Satellite Centre, Bangalore 560 017, India \\ e-mail: \{goel, mssiva\}@isac.ernet.in
}

\begin{abstract}
Remote sensing satellites are required to meet stringent pointing and drift rate requirements for imaging operations. For achieving these pointing and stability requirements, continuous and accurate three-axis attitude information is required. Inertial sensors like gyros provide continuous attitude information with better short-term stability and less random errors. However, gyro measurements are affected by drifts. Hence over time, attitudes based on the gyro reference slowly diverge from the true attitudes. On the other hand, line-of-sight (LOS) sensors like horizon sensors provide attitude information with long-term stability. Their measurements however are affected by the presence of random instrumental errors and other systematic errors. The limitations of inertial and line-of-sight sensors are mutually exclusive. Hence, by optimal fusion of attitude information from both these sensors, it is possible to retain the advantages and overcome the limitations of both, thereby providing the precise attitude information required for control.

This paper describes an improved earth-pointing scheme by fusion of the threeaxis attitude information from gyros and horizon sensor roll and pitch measurements along with yaw updates from the digital sun sensor. A Kalman Filter is used to estimate the three-axis attitude by online estimation and corrections of various errors from the sensor measurements. Variations in orbit rate components are also accounted for using spacecraft position and velocity measurements from the satellite positioning system. Thus precise earth-pointing is achieved.
\end{abstract}

Keywords. Attitude control; imaging spacecraft; Kalman Filter.

\section{Introduction}

With increase in user requirements of satellite imagery with better than $1 \mathrm{~m}$ accuracy, the pointing specifications of earth-pointing satellites, which are mainly used for resource management, oceanography and cartographic applications, are becoming more stringent and they are of the order of $0 \cdot 01$ to $0.05^{\circ}$. Further to improve the location and targeting accuracies of 
the imagery, the internal distortion needs to be better than $1 \mathrm{~m}$. To achieve this, the spacecraft rate variations during imaging time is required to be better than $5 \cdot 0 \times 10^{-0 \cdot 05} \mathrm{deg} / \mathrm{s}$.

To achieve this tight specifications of pointing and drift rate, accurate information of the three-axis attitude of the spacecraft is required continuously for the controller. Gyros provide three-axis inertial attitude reference. Gyro measurements are available continuously and with better short-term stability and low noise level. However, gyro measurements are affected by inherent drifts which result in slow divergence of measurements over time (Venkateswaran et al 1993). Gyro measurements are also subjected to scale factor and misalignment errors. Hence, gyro measurements used for attitude referencing need updates from line-of sight attitude sensors.

Digital Sun Sensors (DSS) provide the true yaw attitude information at poles, while the Horizon (Earth) sensor provides roll and pitch attitude information. Measurement accuracy of an earth sensor is affected by the presence of random instrumental errors and other systematic errors (Rao \& Padmanabhan 1983). The systematic errors are contributed by earth's oblateness, spacecraft altitude variations, seasonal changes in earth's radiance, ambient temperature and misalignment biases. Also due to orbit geometry, true yaw attitude from DSS is available only at the poles.

In the present work, fusion of attitude information from the DSS, earth sensor and gyro is done in an optimal way using a Kalman Filter which estimates and corrects on-line the various errors of the gyro and earth sensor, thereby providing accurate three-axis information for the controller. For nadir pointing, it is required to control the spacecraft with respect to the instantaneous orbit reference frame. Hence, information regarding actual orbit rate variations caused by eccentricity are required to determine the attitude control signal with respect to the desired orbit reference frame. Both DSS and earth sensor provide attitude reference in the earth pointing (instantaneous) orbit reference frame. But as gyros provide inertial reference, gyro measurements have to be expressed in orbit reference frame. In earlier IRS satellites, average orbit pitch rate was used to propagate the gyro-based attitude in earth-pointing frame between updates by DSS and ES.

From the on-orbit experience, orbit rate variations are observed due to eccentricity (Nagarajan \& Jayashree 2002) with components along all three axes. Variations of the order of $2.0 \times 10^{-04} \mathrm{deg} / \mathrm{s}$ along pitch and $2.0 \times 10^{-05} \mathrm{deg} / \mathrm{s}$ along yaw were observed. If these variations are not accounted for in deriving the earth-pointing attitude information, spacecraft pointing is affected by about $0 \cdot 2^{\circ}$. Also, these variations are found to possess harmonics of orbit frequency component. Even though fundamental harmonic component of pitch can be estimated, yaw variation and other harmonics of orbit rate cannot be estimated due to observability problem. In this work, orbit position and velocity measured by SPS (satellite positioning system) is used to derive the orbit rate components and they are accounted in determining the earth-pointing attitude (Control Systems Group 2002).

\section{Design of gyro-based attitude reference scheme with horizon sensor updates}

Various modules involving in horizon sensor updated gyro reference scheme are described below.

\subsection{Gyro measurements for attitude reference}

Inertial reference unit (IRU) used for attitude reference consists of three orthogonally mounted dynamically tuned gyros (DTG). A DTG is a two-degree of freedom gyro in which the rotor 


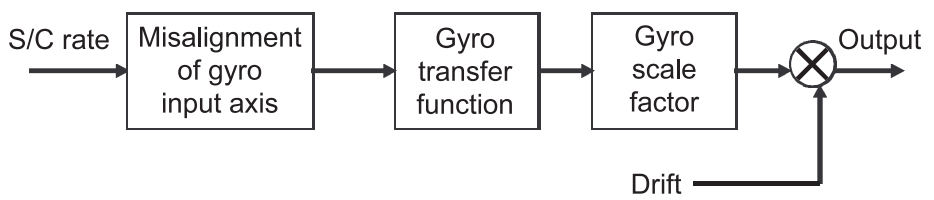

Figure 1. Block scheme of gyro measurement simulation.

is supported from the shaft through a hook's joint suspension. The gyro consists of four major subassemblies, namely, the case, the pick off, the torque coils and the rotor together with the suspension system. The pick-off and torque coils control the attitude of the rotor relative to the gyro case based on the law of precession.

When an input rate is applied to the gyro case, the case fixed pick-off senses the change of rotor attitude, relative to the case and causes the caging loop to provide the necessary torque to the torque motors in a was so as to reduce this change to zero. The current through this re-balancing torque coils is the measure of the spacecraft rate fed at the gyro case.

Gyro integrates the instantaneous spacecraft rate between two sync pulses (i.e. over $32.768 \mathrm{~ms}$ ) received from AOCE and provides the incremental angle outputs to AOCE. Gyro measurements are subjected to the following errors, namely drifts, scale factor errors, misalignment of gyro input axes and random errors in drift, and quantization error in output. All these errors have to be eliminated from the measurement to get the true attitude reference for pointing.

The block schematic of gyro measurement is shown in figure 1 .

\subsection{Horizon sensor modelling}

A conical scanning earth sensor used in low earth orbit satellites like IRS (Indian Remote Sensing) is considered. This sensor views the earth in 14-16 micrometre $\mathrm{CO}_{2}$ absorption band of infrared radiation. The sensor employs a rotation mechanism which creates a conical scan of space by the field-of-view (Wertz 1986). When the scan crosses the horizon (from space-to-earth and vice versa), it detects the change in radiance between space and the earth as shown in figure 2 .

Spacecraft roll and pitch errors are computed using the timing information of these crossings, standard chord width and a reference pulse generated by sensor electronics (see figure 3).

As for the error sources of the horizon sensor, the oblate shape of earth and the latitude and season-dependent variation of radiance are the dominants. The earth's oblateness leads to systematic error with twice the orbital frequency, whereas radiance change over latitudes gives systematic error with orbital frequency. Hence, roll and pitch systematic errors are modelled as sinusoidal with harmonics of orbital frequency (Alex \& Shrivastava 1989).

\subsection{Digital sun sensor yaw update}

The digital sun sensor (Sidi 1997) consists of an optical head having a quartz block with a slit pattern on one surface and a gray-coded pattern on the opposite surface. Light falling on the block, passes through the slit and illuminates the gray-coded pattern and the corresponding solar cells generate electrical output. The electrical output is converted to digital patterns, 


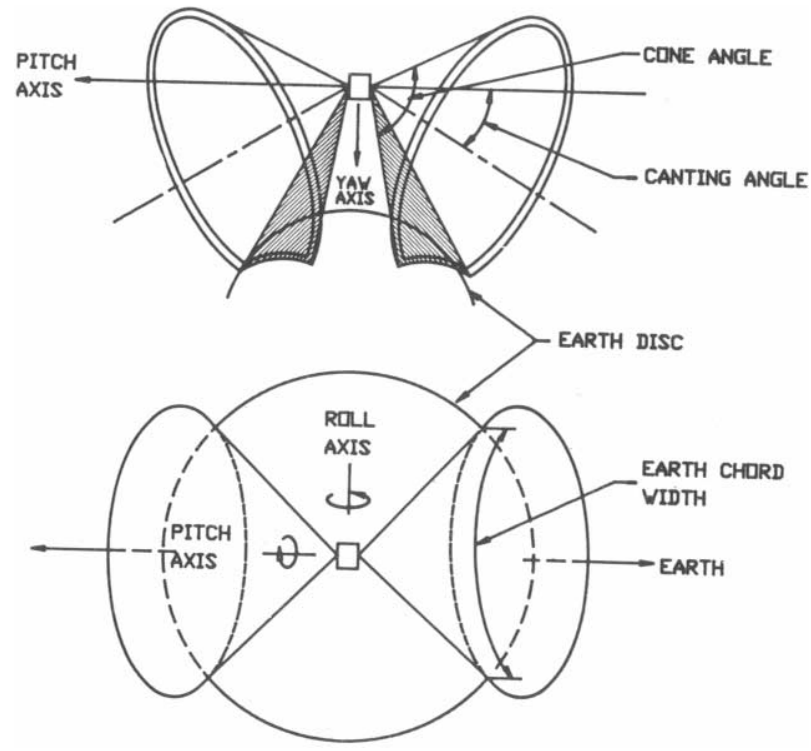

Figure 2. Conical scanning sensor operating principle.

thus a gray-coded output is formed. This electrical or gray-coded output depends on the sun aspect angle.

This sun-aspect angle is a measure of S/C yaw attitude when the sun is in roll-pitch plane. Thus the DSS can measure the true yaw error at poles, where the sun lies in roll-pitch plane.

\subsection{Gyro data processing}

Processing of gyro data to extract the inertial reference for $\mathrm{S} / \mathrm{C}$ attitude involves the following steps.

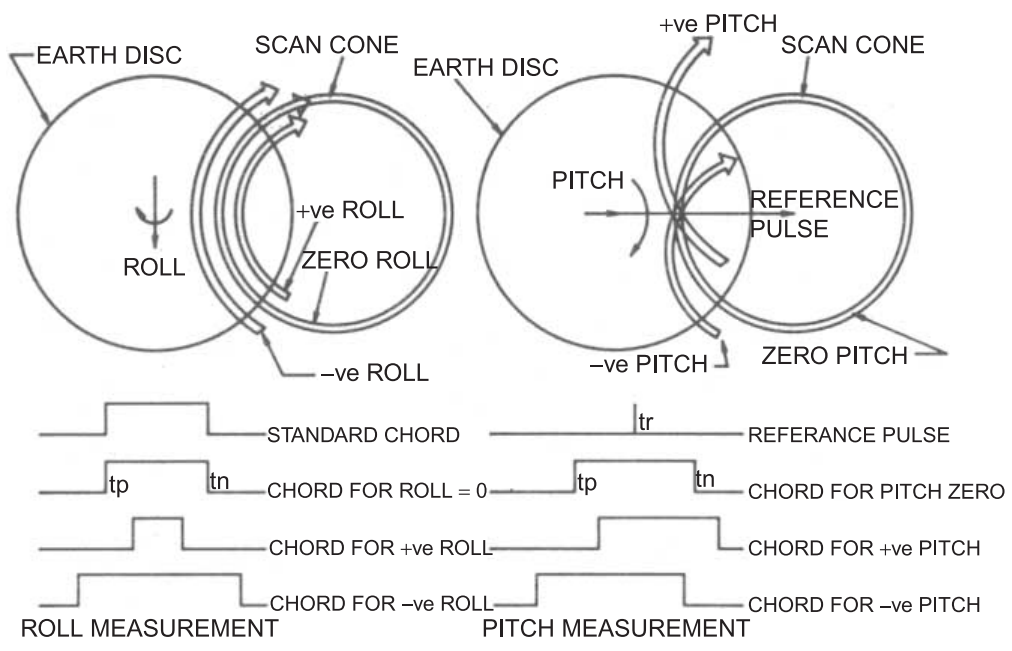

Figure 3. Roll and pitch error measurement. 
(1) Drift rate compensation is performed on the measured gyro incremental angles based on the gyro drifts determined by ground tests and using gyro calibration exercises. This is performed in gyro frame.

(2) Scale factor error correction is performed on the drift corrected gyro output to get the actual gyro measurement of attitude change in gyro frame. Scale factor correction is performed using uplinked correction parameters which in turn are computed by ground test and estimation schemes.

(3) Actual change in spacecraft attitude is obtained from this error corrected angles by correcting for the gyro input axis misalignment. Misalignment parameters are determined using ground tests before launch and estimation schemes with special calibration exercises during on-orbit.

With all these corrections, near-true inertial attitude of the spacecraft is obtained.

\subsection{Fusion of gyro and attitude sensor data}

Even though gyro measurements are corrected for pre-determined error sources, uncorrected error variations in drift, scale factor and misalignment still remain in gyro output and they contribute an equivalent drift component in measured attitude during nadir imaging. Using the attitude information from DSS and earth sensor, the resultant gyro drift in body frame is estimated in this scheme and corrected from the gyro output.

Similarly, dominant components of earth sensor systematic error, namely twice the orbit frequency component in roll and pitch output are estimated and corrected from the sensor measurement. The other components of systematic errors are corrected, based on the ground predicted values.

Kalman Filter (Pillai 1980) is used for estimation of these errors and provides error-free attitude estimate of the spacecraft.

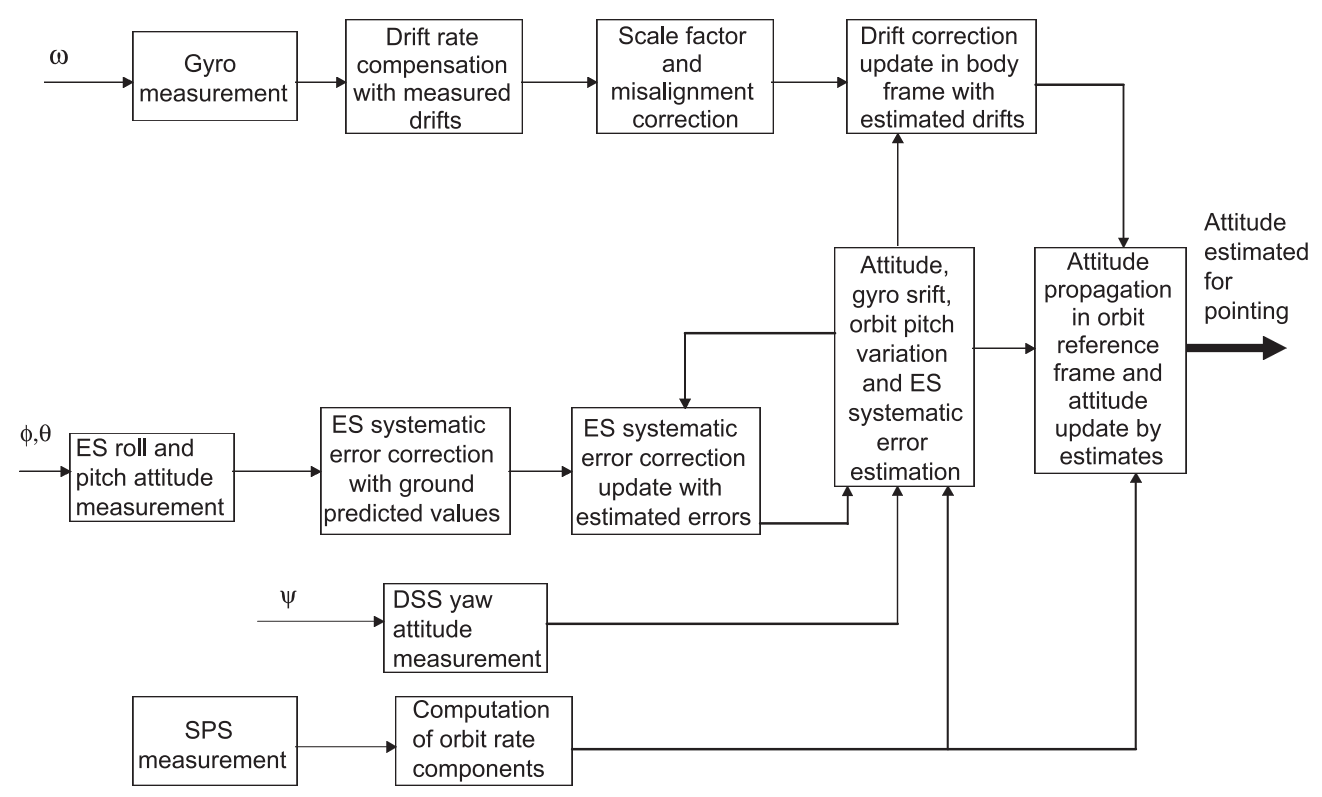

Figure 4. Functional block scheme of horizon sensor updated gyro reference scheme. 
Orbit information from SPS measurements are used to propagate the gyro referenced attitude in between LOS updates for getting attitude estimate with respect to instantaneous orbit reference frame.

The overall block scheme of gyro-based attitude reference system with DSS and horizon sensor update is shown in figure 4.

\subsection{Usage of orbit information from SPS}

The satellite positioning system (SPS) measures the spacecraft position and velocity in an earth-centred earth-fixed (ECEF) reference frame. Using the knowledge of sidereal angles, the measured position and velocity vectors are expressed in earth-centred inertial (ECI) frame. Using these two vectors, the quaternion that describes the instantaneous position of the spacecraft orbit with respect to the ECI frame is obtained. The relative change in this quaternion between two gyro measurements is used to propagate the gyro-based attitude estimate in orbit reference frame in between the updates from line-of-sight sensors. There is also provision to estimate the variations in pitch component of orbit rate, which is used for deriving the earth-pointing attitude in case of non-availability of SPS measurement.

\section{Estimation using Kalman Filter}

\subsection{Attitude kinematics}

For a three-axis stabilized earth pointing spacecraft, the yaw $(\psi)$, roll $(\phi)$ and pitch $(\theta)$ angles follow the well-known linearized kinematic differential equations,

$$
\begin{aligned}
& \dot{\psi}=-\omega_{0 Z} \phi+\omega_{0 X}+\omega_{1}, \\
& \dot{\phi}=\omega_{0 Z} \psi+\omega_{0 Y}+\omega_{2}, \\
& \dot{\theta}=\omega_{0 Z}+\omega_{3},
\end{aligned}
$$

where $\omega_{1}, \omega_{2}, \omega_{3}$ are the yaw, roll and pitch body rates and $\omega_{0 X}, \omega_{0 Y}$ and $\omega_{0 Z}$ are the yaw, roll and pitch components of orbit rate. Assuming that the gyro outputs contain drift and noise, the above equations can be written as

$$
\begin{aligned}
& \dot{\psi}=-\omega_{0 Z} \phi+\omega_{0 X}+\omega_{g X}-b_{X}-\eta_{X}, \\
& \dot{\phi}=\omega_{0 Z} \psi+\omega_{0 Y}+\omega_{g Y}-b_{Y}-\eta_{Y}, \\
& \dot{\theta}=\omega_{0 Z}+\omega_{g Z}-b_{Z}-\eta_{Z},
\end{aligned}
$$

where, $\omega_{g X}, \omega_{g Y}, \omega_{g Z}$ are the yaw, roll and pitch gyro rate outputs. $b_{X}, b_{Y}$ and $b_{Z}$ are the drifts and $\eta_{X}, \eta_{Y}$ and $\eta_{Z}$ are the rate noise (white Gaussian) components of yaw, roll and pitch gyros respectively. Thus, using gyros in model replacement mode eliminates the necessity for elaborate spacecraft dynamic modelling, which is associated with uncertainties of control and disturbance torques and spacecraft parameters. It also offers very high accuracy.

\subsection{Gyro residual drifts}

Gyro drifts are usually modelled as fixed and random. Typical random drift analysis of dynamically tuned gyros (Sidi 1997) shows that the outputs contain white noise drift rate, negligible 
random walk component and a correlated bias with very large correlation time $(10 \mathrm{~h})$. Since the attitude updates from LOS sensors are available more frequently, it is reasonable to neglect the correlated drifts and it is sufficient to consider only the fixed drifts and rate noise. The fixed drifts can be modelled as given below.

$$
\dot{b}_{X}=0 \cdot 0, \dot{b}_{Y}=0 \cdot 0 \text {, and } \dot{b}_{Z}=0 \cdot 0 \text {. }
$$

\subsection{Orbit variation and ES systematic error components}

Orbit pitch rate component $\omega_{0 Z}$ can be expressed as

$$
\omega_{0 Z}=\omega_{0} \times\left[1 \cdot 0+e \times \sin \left(\omega_{0} \times t\right)\right]=\omega_{0}+\omega_{0 E},
$$

where $\omega_{0}$ is the nominal orbit rate, $e$ is the eccentricity of the orbit and $\omega_{0 E}$ is the variation in orbit rate along pitch due to eccentricity.

Table 1. Parameters used in simulation.

Spacecraft orbit parameters

Semi-major axis $(a)$

Eccentricity $(e)$

Inclination $(i)$

Argument of latitude $(\omega)$

Right ascension of ascending node $(\Omega)$

Mean anomaly $(m)$

Orbit epoch

Orbit period

Spacecraft moment of inertia

Along yaw, roll and pitch

Wheel capacity

Gyro parameters

Fixed drifts along yaw, roll and pitch

Random drift

Quantization noise in incremental angle

Gyro update interval

Digital sun sensor parameters

Sensor noise

DSS update interval

Earth sensor parameters

Sensor measurement noise

Sampling rate

Systematic error $2 \omega_{0}$ component

Along roll

Along pitch
$7204.0861827 \mathrm{~km}$

0.0022043

98.6839699

$113 \cdot 1045383$

$78 \cdot 1290476$

$66 \cdot 7689358$

$2002 / 1 / 1 / 3 / 50 / 0 / 0$

$6080.929000 \mathrm{~s}$

1920,1419 and $1025 \mathrm{~kg}-\mathrm{m}^{2}$

$5 \mathrm{NMS}, 0.05 \mathrm{Nm}$

$5 \cdot 0,-5 \cdot 0,5 \cdot 0 \mathrm{deg} / \mathrm{h}$

$0.15 \mathrm{deg} / \mathrm{h}(3 \sigma)$

4 bit $\left(6 \cdot 25 \times 10^{-05} \mathrm{deg}\right)(3 \sigma)$

$32.768 \mathrm{~ms}$

$0.03 \operatorname{deg}(3 \sigma)$

Once in orbit (at South Pole)

$0 \cdot 12 \operatorname{deg}(3 \sigma)$

$4 \mathrm{~Hz}$

$0 \cdot 2 \mathrm{deg}$

$0.3 \mathrm{deg}$ 

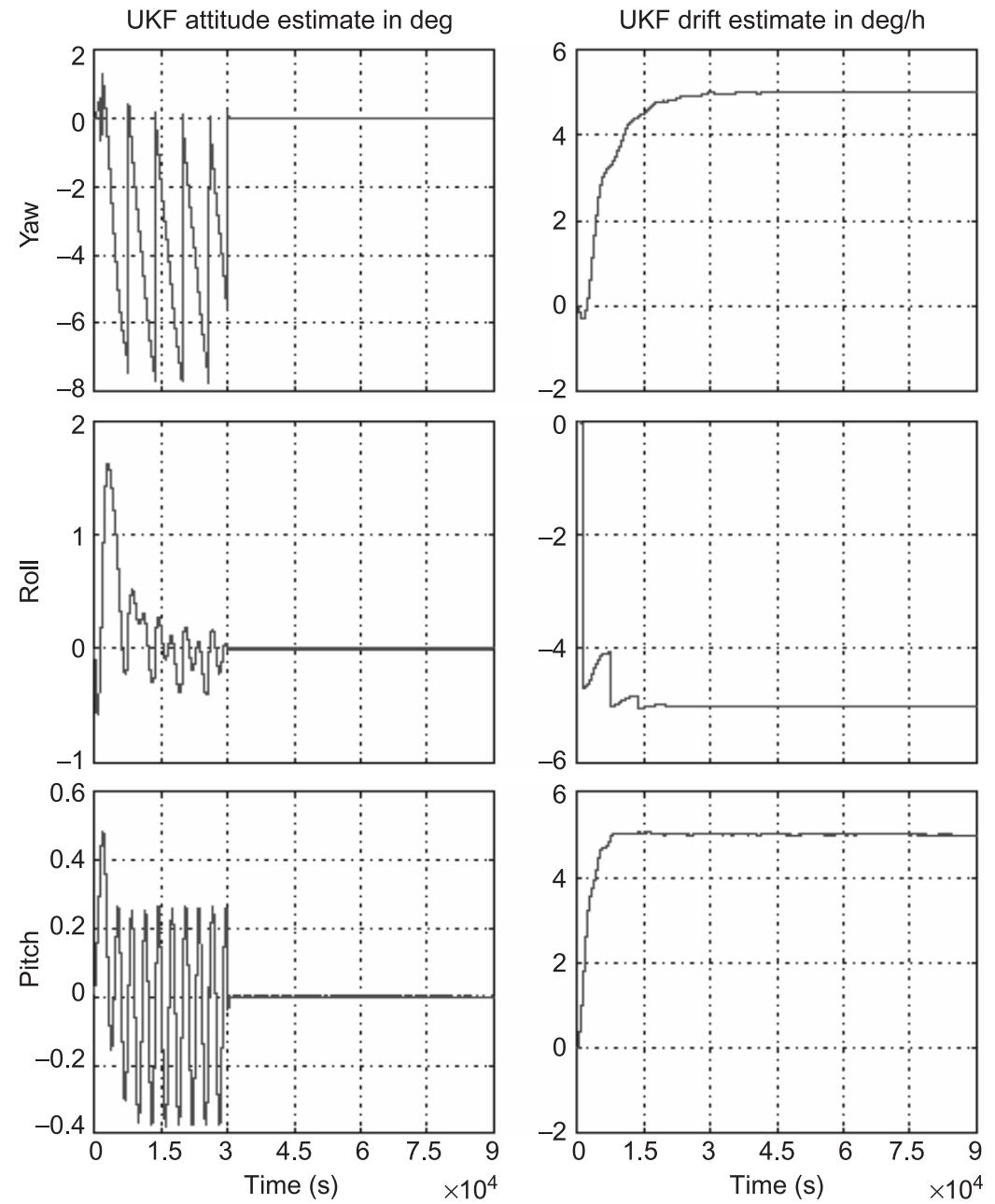

Figure 5. Estimation of S/C attitude and gyro drifts using ES-based Kalman Filter - Kalman Filter in control loop from $30,000 \mathrm{~s}$.

Similarly, twice the orbit frequency components of ES systematic error along roll and pitch are modelled as

$$
\phi_{e}=\phi_{m} \times \sin \left(2 \times \omega_{0} \times t\right) \text { and } \theta_{e} \theta_{m} \times \sin \left(2 \times \omega_{0} \times t\right) .
$$

\subsection{Kalman Filter model}

System dynamics for the estimation problem is obtained by annexing (3), (4) and (5) to the kinematic equation (2) to arrive at a 12th state augmented state model as given below.

$$
\dot{X}=A X+B U+G \eta,
$$

where $X=\left\{\psi, \phi, \theta, b_{X}, b_{Z}, \omega_{0 E}, \dot{\omega}_{0 E}, \phi_{E}, \dot{\phi}_{E}, \theta_{E}, \dot{\theta}_{E}\right\}$ are the states of the estimator and $U=\left\{\omega_{1}+\omega_{0 X}, \omega_{2}+\omega_{0 Y}, \omega_{3}+\omega_{0 Z},\right\}$ are the process inputs and the matrices $A, B$ and $G$ are derived from (2) to (5). 

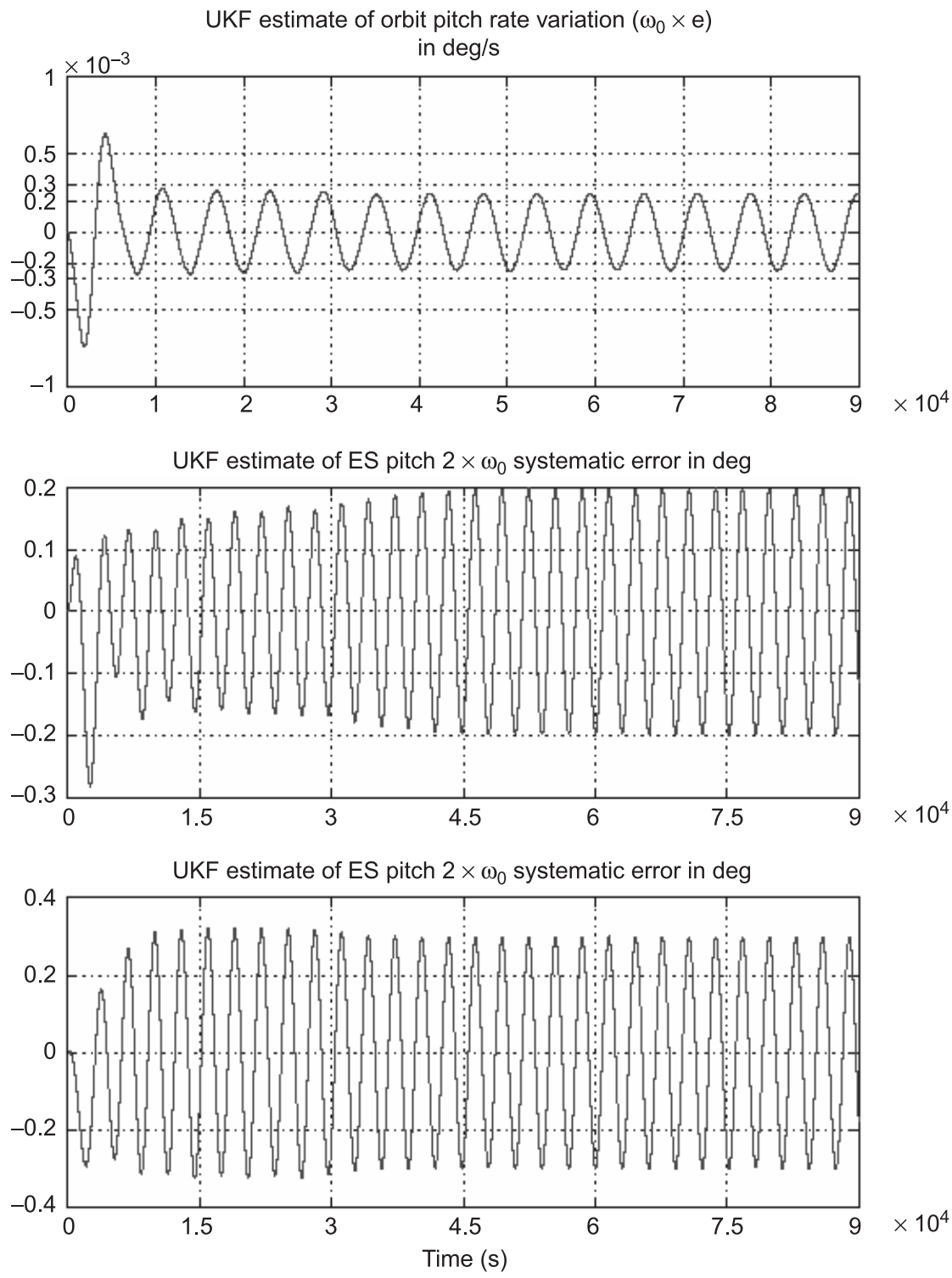

Figure 6. Estimation of orbit pitch variation and ES systematic errors using ES based-Kalman Filter - Kalman Filter in control loop from 30,000 s.

The measurement equation for this system can be written in the standard form

$$
Z=H x+V
$$

where $Z=\left(Z_{1}, Z_{2}, Z_{3}\right)^{T}$ is the measurement vector whose components $Z_{1}, Z_{2}$ and $Z_{3}$ are the digital sun sensor yaw, earth sensor roll and earth sensor pitch output respectively, and the vector $V=\left[V_{1}, V_{2}, V_{3}\right]^{T}$ refers to respective measurement noise for these sensors and $H$ is the measurement matrix. 

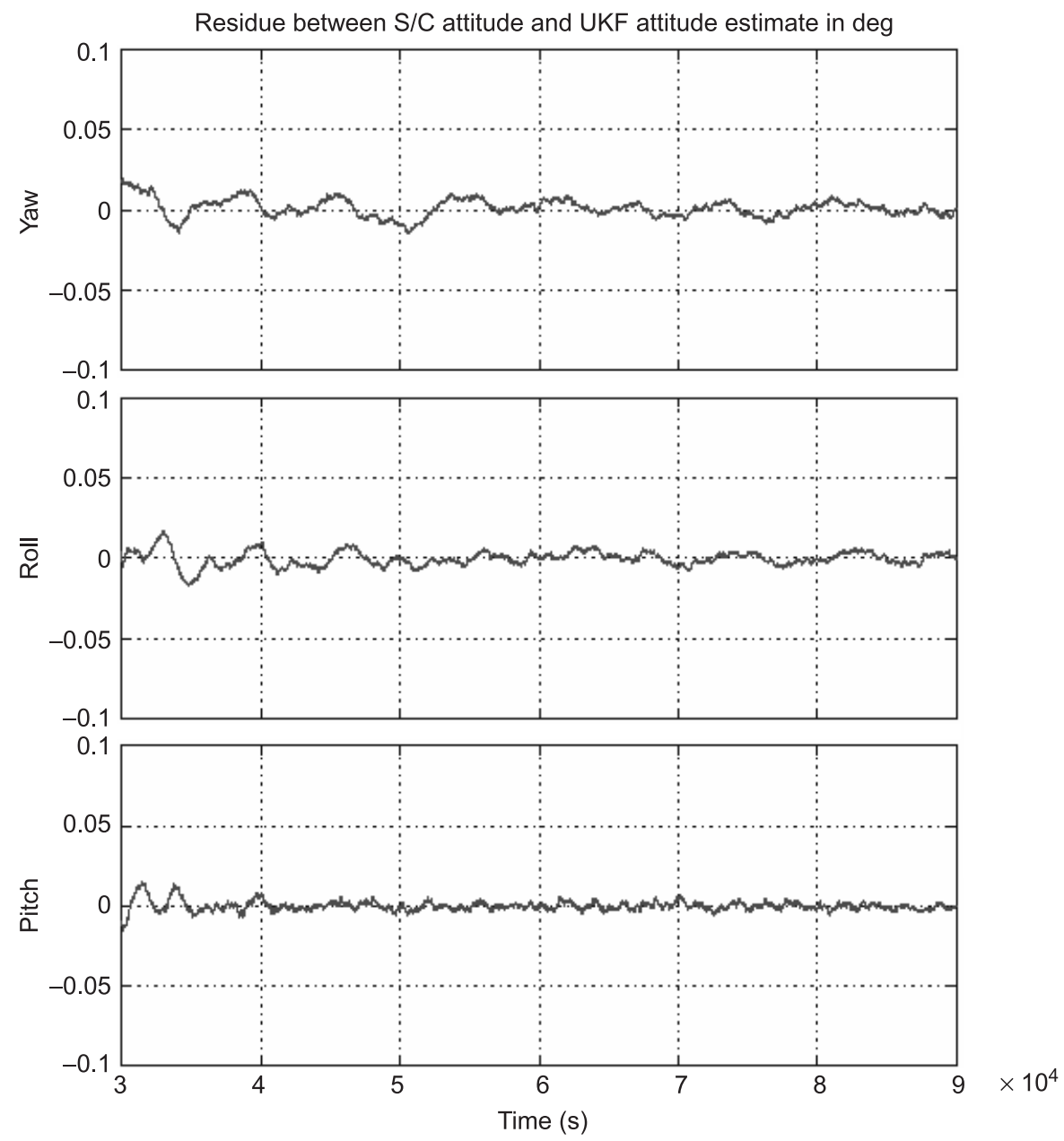

Figure 7. Steady state attitude estimation error of ES-based Kalman Filter.

\subsection{Observability}

The system described by (6) and (7) is fully observable, i.e., when yaw measurement is possible. But, when yaw is not available due to unfavourable geometry and spacecraft eclipse, roll gyro drift is not observable (Control Systems Division 1987). However, it can be seen that the response due to unobservable mode is bounded in nature during these periods and the roll drift estimation error converges to zero asymptotically with further yaw updates. Also, since the gyro drifts are fairly constant over these short periods between yaw updates, the effect on estimation of other parameters, including earth sensor systematic errors, are negligible.

\section{Simulation results}

To analyse the performance of this, precision pointing reference system, simulations were carried out for a typical remote sensing satellite. The parameters used in simulation are 

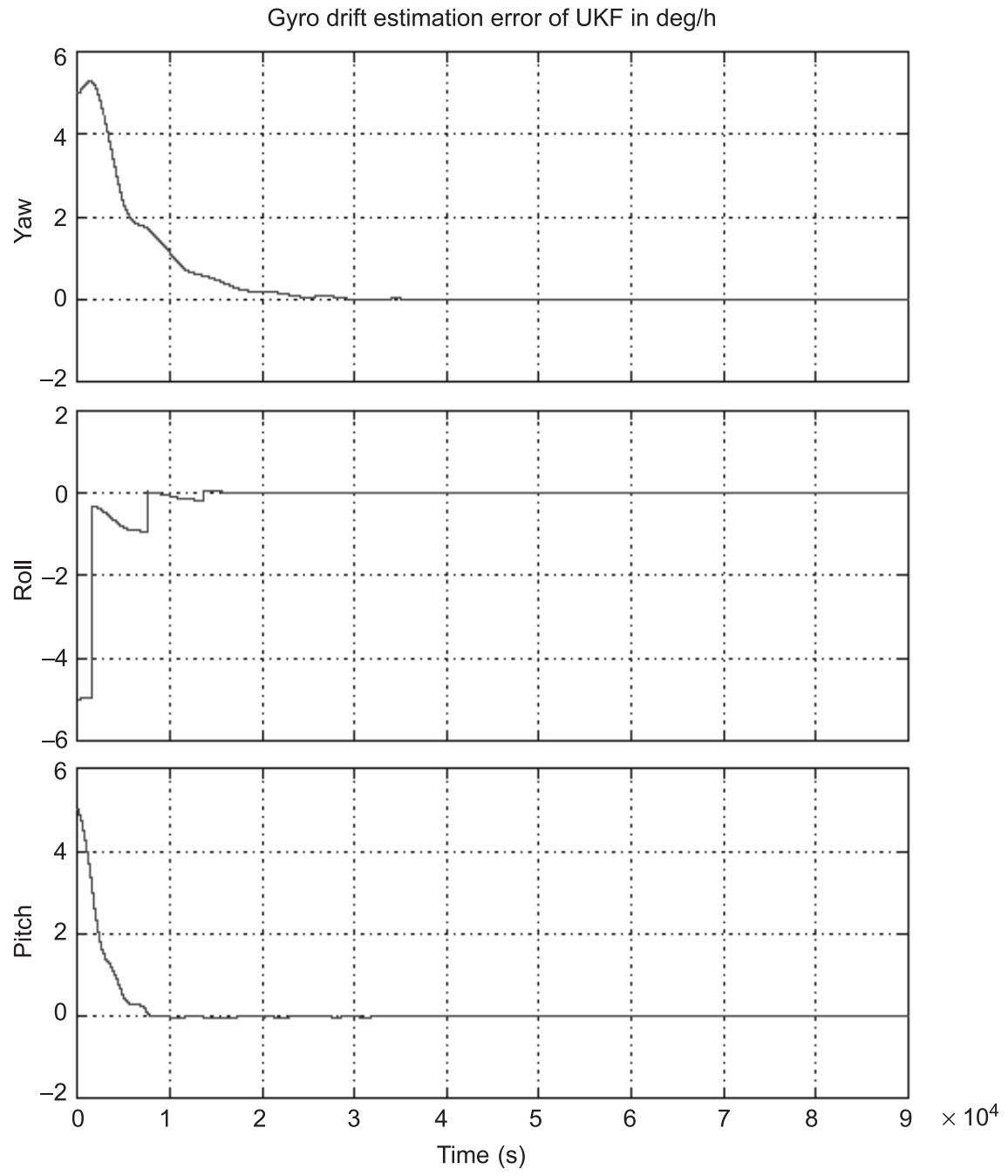

Figure 8. Gyro estimation error of Es-based Kalman Filter.

summarized in table 1. Simulations were carried out for durations of about 15 orbits (period of each orbit is 6080.929 s). During the first 30,000 seconds, the spacecraft was controlled with direct measurements of DSS, earth sensor (ES) and gyro. Later, the Kalman Filter scheme which performs the optimal fusion of DSS, ES and gyro outputs along with SPS data is selected for control action. As during nadir pointing with uniform rate, the gyro scale factor and misalignment errors contribute additional drift, only the drift error of gyro is considered for simulation.

Figures 5 and 6 show the open loop and closed loop performance of earth sensor-based Kalman Filter in estimating the S/C attitude, gyro drift and earth sensor systematic errors. The Kalman Filter corrects these errors from measurement and provides a near-true S/C attitude information for control action. This is clear as seen from figure 7 which shows the closeness of attitude estimate to the actual $\mathrm{S} / \mathrm{C}$ attitude. Estimation error is better than $0.02^{\circ}$. Figure 8 


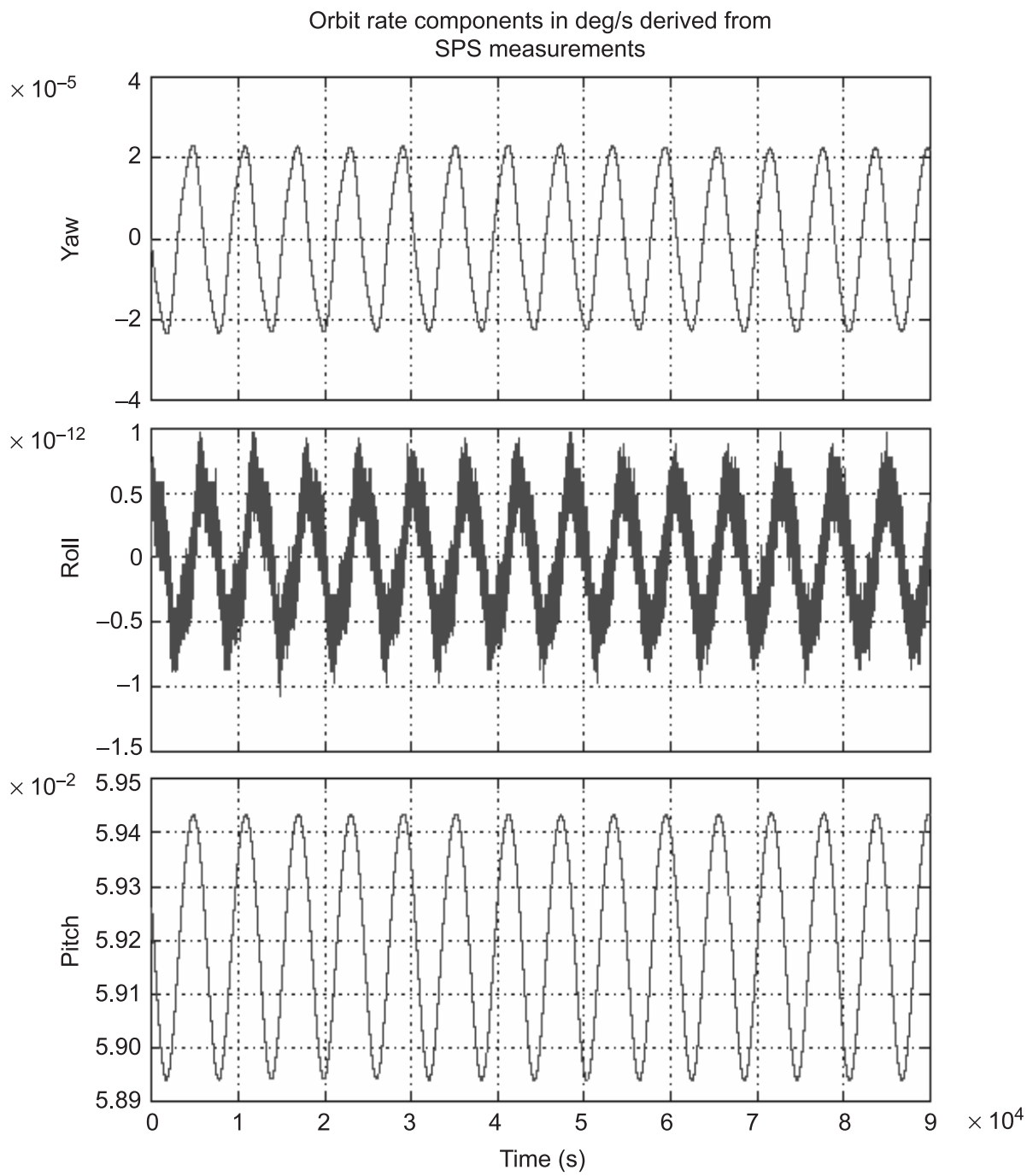

Figure 9. Orbit rate variations along S/C axes derived from SPS measurements.

shows the residual drift after compensating the gyro output with UKF drift estimate. This residual drift is within $0.05 \mathrm{deg} / \mathrm{h}$ during steady state. Figure 9 shows the orbit rate components derived from the SPS measurements. From figures 6 and 9 it is clear that the Kalman Filter estimates the variations in orbit pitch rate exactly.

Figure 10 shows the S/C pointing performance with Kalman Filter in control loop and the pointing error is found to be of the order of 0.01 to $0.02^{\circ}$. Figure 11 show the drift rate performance of the spacecraft with Kalman Filter in loop. Drift rate is well within $5 \cdot 0 \times 10^{-05} \mathrm{deg} / \mathrm{s}$.

\section{Conclusion}

A novel scheme, which performs an optimal fusion of attitude information from gyros, digital sun sensor and earth sensor along with the orbit information from the satellite positioning 

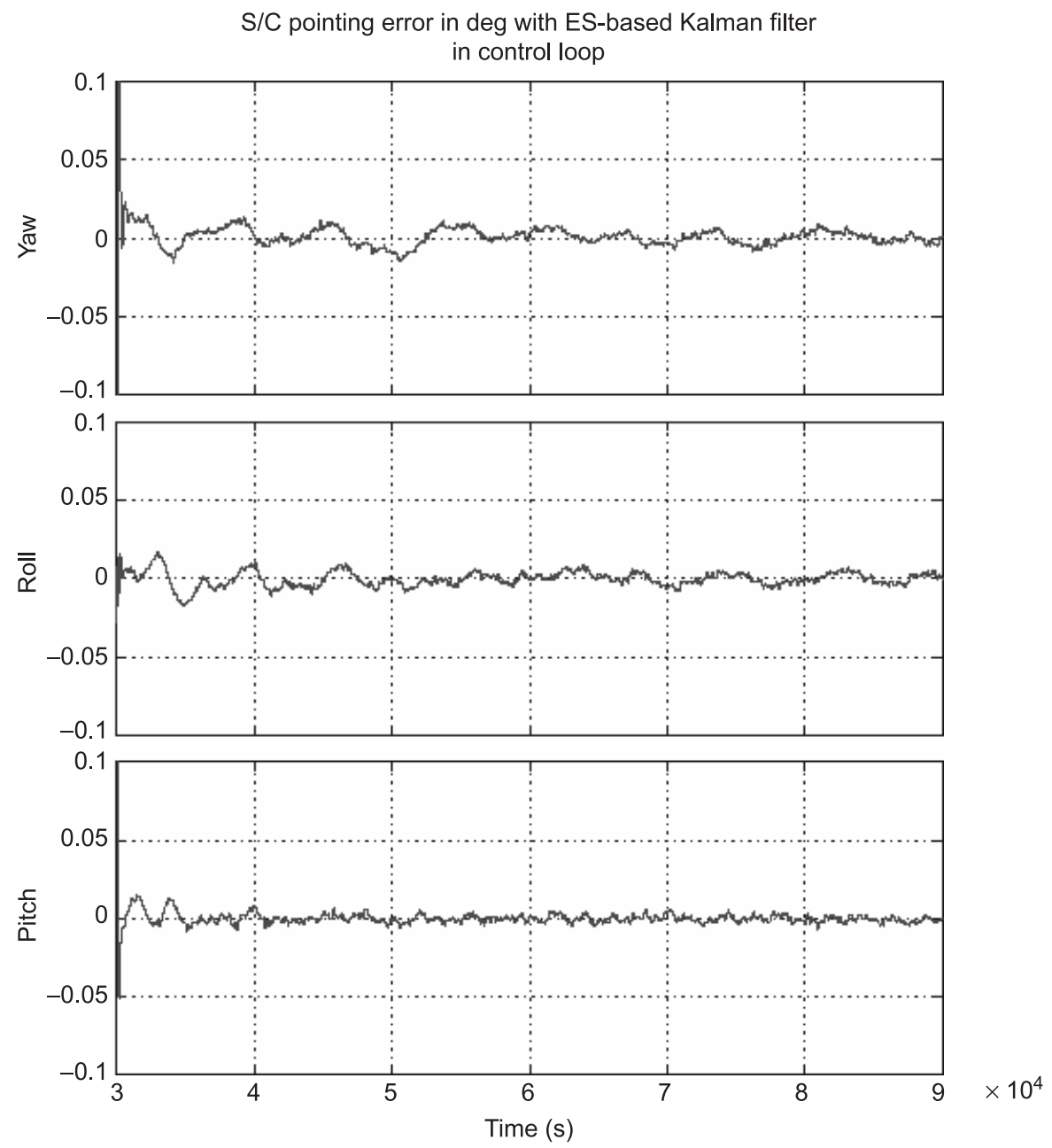

Figure 10. S/C pointing performance with fusion of ES and gyro information of attitude and SPS measurement orbit position.

system, to provide a true attitude reference for precision pointing of remote sensing satellites is presented. Simulations carried out with this scheme show improved pointing and drift rate performance, which is a major requirement for the resource management or cartographic mission.

This scheme of optimal fusion is the best option for a nadir pointing spacecraft. For spacecraft with stereo imaging, spot imaging or high resolution strip imaging, the effect of gyro scale factor and misalignment plays a major role in influencing the pointing reference, since such applications require varying rates along all the three axes of the spacecraft. Even though ground calibration schemes are available for the determination of these errors, on-board estimation and correction of these scale factor and misalignment errors is the best option for improving the pointing reference. Future work in precision pointing should be along this direction. 

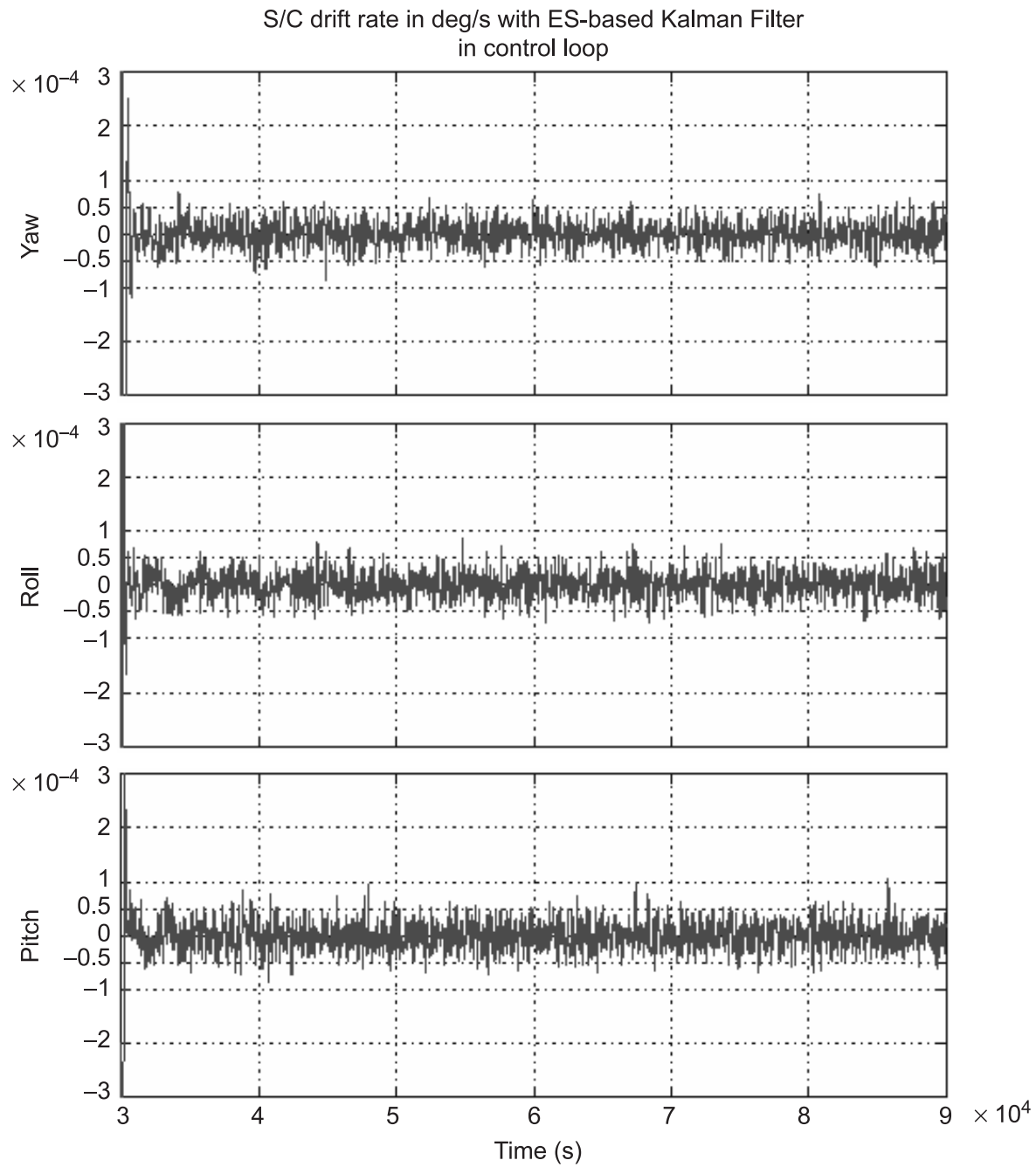

Figure 11. S/C drift rate performance with fusion of ES and gyro information of attitude and SPS measurement orbit position.

\section{References}

Alex T K, Shrivastava S K 1989 On-board correction of systematic errors of scanning infrared earth sensor. IEEE Trans. Aerosp. Electron. Syst. 25: 373-379

Control Systems Division 1987 Update Kalman Filter for onboard attitude determination. Technical Report No. ISAC-21-87-12-05-11, ISRO Satellite Centre, Bangalore

Control Systems Group 2002 New and modified onboard AOCS software requirements, algorithm designs for IRS P6. Doc No. ISRO-ISAC-IRS-P6-PR-0343, ISRO Satellite Centre, Bangalore

Sidi M J 1997 Spacecraft dynamics and control (Cambridge: University Press)

Nagarajan N, Jayashree M S 2002 On motion of the eccentricity vector for IRS-1A and IRS-1B. J. Spacecr. Technol. 2(2): 44-51

Pillai S K 1980 The Kalman Filter - A brief survey. Technical report ISRO-SHAR-TR-84-80, Satish Dhawan Satellite Centre, Sriharikota 
Rao H R, Padmanabhan P 1983 Effect of earth's oblateness in roll and pitch measurements using conical horizon scanners for IRS. Technical Report IRS-ISAC-42-83-06-05-001, ISRO Satellite Centre, Bangalore

Venkateswaran N, Krishnakumar E, Goel P S, Seetharam Bhat M 1993 Star sensor/earth sensor updated gyro reference for precision pointing spacecraft. J. Spacecr. Technol. 3(2): 25-37

Wertz J R 1986 Spacecraft attitude determination and control (Dordrecht: Reidel) 